\title{
A note on the Diophantine equation $a^{x}+b^{y}=c^{z}$
}

\author{
by \\ Zhenfu Cao (Harbin)
}

1. Introduction. Let $\mathbb{Z}, \mathbb{N}, \mathbb{P}$ be the sets of integers, positive integers and primes respectively, and

$$
\mathbb{P}^{\mathbb{N}}=\left\{p^{n} \mid p \in \mathbb{P} \text { and } n \in \mathbb{N}\right\} .
$$

Clearly, $\mathbb{P} \subseteq \mathbb{P}^{\mathbb{N}}$. In [13] Nagell first proved that if $\max (a, b, c) \leq 7$, then all the solutions $(x, y, z) \in \mathbb{N}^{3}$ of the equation

$$
a^{x}+b^{y}=c^{z}, \quad a, b, c \in \mathbb{P}, a>b
$$

are given by

$$
\begin{aligned}
& (a, b, c)=(3,2,5):(x, y, z)=(1,1,1),(2,4,2) ; \\
& (a, b, c)=(5,2,3):(x, y, z)=(2,1,3),(1,2,2) ; \\
& (a, b, c)=(5,3,2):(x, y, z)=(1,1,3),(1,3,5),(3,1,7) \\
& (a, b, c)=(3,2,7):(x, y, z)=(1,2,1) \\
& (a, b, c)=(7,2,3):(x, y, z)=(1,1,2),(2,5,4) ; \\
& (a, b, c)=(7,3,2):(x, y, z)=(1,2,4) ; \\
& (a, b, c)=(5,2,7):(x, y, z)=(1,1,1) \\
& (a, b, c)=(7,5,2):(x, y, z)=(1,2,5) .
\end{aligned}
$$

Later, Mąkowski [11], Hadano [7], Uchiyama [23], Qi Sun and Xiaoming Zhou [16], and Xiaozhuo Yang [24] determined all solutions $(x, y, z) \in \mathbb{N}^{3}$ of equation (1), when $11 \leq \max (a, b, c) \leq 23$. In [1] we have given all solutions $(x, y, z) \in \mathbb{N}^{3}$ of equation (1), when $29 \leq \max (a, b, c) \leq 97$ (60 solutions in total), and we have proved the following:

TheOrem A. If $\max (a, b, c)>13$, then equation (1) has at most one solution $(x, y, z) \in \mathbb{N}^{3}$ with $z>1$.

1991 Mathematics Subject Classification: Primary 11D61.

Key words and phrases: exponential Diophantine equation.

Supported by the National Natural Science Foundation of China and the Heilongjiang Provincial Natural Science Foundation. 
A more general result was given in [2]. Let $A, B \in \mathbb{N}, A>B>1$ and $\operatorname{gcd}(A, B)=1$. If the equation

(2) $X^{2}+A B Y^{2}=p^{Z}, \quad X, Y, Z \in \mathbb{N}, p \in \mathbb{P}$, and $\operatorname{gcd}(X, Y)=1$,

has a solution $(X, Y, Z)$, then there exists a unique solution $\left(X_{p}, Y_{p}, Z_{p}\right)$ which satisfies $Z_{p} \leq Z$, where $Z$ runs over all solutions of (2). That $\left(X_{p}, Y_{p}, Z_{p}\right)$ is called the least solution of (2). From [2] we have

Theorem B. If $x, y \in \mathbb{N}$ satisfy the equation

$$
A x^{2}+B y^{2}=2^{z}, \quad z>2,\left.x\right|^{*} A,\left.y\right|^{*} B,
$$

where the symbol $\left.x\right|^{*} A$ means that every prime divisor of $x$ divides $A$, then

$$
\left|A x^{2}-B y^{2}\right|=2 X_{2}, \quad x y=Y_{2}, 2 z-2=Z_{2},
$$

except for $(A, B, x, y, z)=(5,3,1,3,5),(5,3,5,1,7)$ and $(13,3,1,9,8)$.

Theorem C. If $x, y \in \mathbb{N}$ satisfy the equation

$$
A x^{2}+B y^{2}=p^{z}, \quad p \in \mathbb{P},\left.x\right|^{*} A,\left.y\right|^{*} B,
$$

then

$$
\left|A x^{2}-B y^{2}\right|=X_{p}, \quad 2 x y=Y_{p}, 2 z=Z_{p}
$$

or

$$
\left|A x^{2}-B y^{2}\right|=X_{p}\left|X_{p}^{2}-3 A B Y_{p}^{2}\right|, \quad 2 x y=Y_{p}\left|3 X_{p}^{2}-A B Y_{p}^{2}\right|, 2 z=3 Z_{p},
$$

the latter occurring only for

$$
A x^{2}+B y^{2}=3^{4 s+3}\left(\frac{3^{2 s}-1}{8}\right)+\left(\frac{3^{2 s+2}-1}{8}\right)=\left(\frac{3^{2 s+1}-1}{2}\right)^{3}=p^{z},
$$

where $s \in \mathbb{N}$.

From Theorems B and C, we have (cf. Lemma 6 of [15])

Theorem D. The equation

$$
a^{x}+b^{y}=c^{z}, \quad \operatorname{gcd}(a, b)=1, c \in \mathbb{P}, a>b>1,
$$

has at most one solution when the parities of $x$ and $y$ are fixed, except for $(a, b, c)=(5,3,2),(13,3,2),(10,3,13)$. The solutions in case $(5,3,2)$ are given by $(x, y, z)=(1,1,3),(1,3,5),(3,1,7)$, in case $(13,3,2)$ by $(1,1,4)$ and $(1,5,8)$, and in case $(10,3,13)$ by $(1,1,1)$ and $(1,7,3)$ (cf. [14], [3]).

In [3], we obtained further results when the right sides of equation (3) and equation (4) are replaced by $4 k^{z}$ and $k^{z}$ respectively, where $k \in \mathbb{N}$.

Recently, N. Terai $[20,21]$ conjectured that if $a, b, c, p, q, r \in \mathbb{N}$ are fixed, and $a^{p}+b^{q}=c^{r}$, where $p, q, r \geq 2$, and $\operatorname{gcd}(a, b)=1$, then the Diophantine equation

$$
a^{x}+b^{y}=c^{z}, \quad x, y, z \in \mathbb{N},
$$


has only the solution $(x, y, z)=(p, q, r)$. The conjecture is clearly false. For example, from Nagell's result [13], we see that the equation $3^{x}+2^{y}=5^{z}$ has two solutions $(x, y, z)=(1,1,1),(2,4,2)$, and the equation $7^{x}+2^{y}=3^{z}$ also has two solutions $(x, y, z)=(1,1,2),(2,5,4)$. Furthermore, if $a=1$ or $b=1$, then the conjecture is also false. So, the condition $\max (a, b, c)>7$ should be added to the hypotheses of the conjecture.

For $p=q=r=2$ the above statement was conjectured previously by Jeśmanowicz. We shall use the term Terai-Jeśmanowicz conjecture for the above conjecture with the added condition that $\max (a, b, c)>7$. Some recent results on the Terai-Jeśmanowicz conjecture are as follows:

(a) If $p=q=r=2$, we may assume without loss of generality that $2 \mid a$. Then we have

$$
a=2 s t, \quad b=s^{2}-t^{2}, \quad c=s^{2}+t^{2},
$$

where $s, t \in \mathbb{N}, s>t, \operatorname{gcd}(s, t)=1$ and $2 \mid s t$. In 1982, we [4] proved that

(Cao.1) if $2 \| s, t \equiv 1(\bmod 4)$, or $2 \| s, t \equiv 3(\bmod 4)$ and $s+t$ has a prime factor of the form $4 k-1$, then the Terai-Jeśmanowicz conjecture holds;

$($ Cao.2) if $(s, t) \equiv(1,6),(5,2)(\bmod 8)$, or $(s, t) \equiv(3,4)(\bmod 4)$ and $s+t$ has a prime factor of the form $4 k-1$, then the Terai-Jeśmanowicz conjecture holds (also see [5], pp. 366-367).

Maohua Le $[8,9,6]$ proved that

(Le.1) if $2 \| s, t \equiv 3(\bmod 4)$ and $s \geq 81 t$, then the Terai-Jeśmanowicz conjecture holds;

(Le.2) if $2^{2} \| a$ and $c \in \mathbb{P}^{\mathbb{N}}$, then the Terai-Jeśmanowicz conjecture holds;

(Le.3) if $t=3, s$ is even, and $s \leq 6000$, then the Terai-Jeśmanowicz conjecture holds.

K. Takakuwa and Y. Asaeda [17-19] considered the case $s=2 s^{\prime}$, $t=3,7,11,15$. For example, they proved that if $2 \nmid s^{\prime}$, then the Terai-Jeśmanowicz conjecture holds.

(b) N. Terai $[20-22]$ considered the cases $(p, q, r)=(2,2,3) ;(2,2,5)$; $(2,2, r)$, where $r \in \mathbb{P}$. He proved that

(Terai.1) if $a=m\left(m^{2}-3\right), b=3 m^{2}-1, c=m^{2}+1$ with $m$ even and $b$ is a prime, and there is a prime $l$ such that $m^{2}-3 \equiv 0(\bmod l)$ and $e \equiv 0$ $(\bmod 3)$, where $e$ is the order of 2 modulo $l$, then equation (5) has only the solution $(x, y, z)=(2,2,3)$;

(Terai.2) if $a=m\left|m^{4}-10 m^{2}+5\right|, b=5 m^{4}-10 m^{2}+1, c=m^{2}+1$ with $m$ even and $b$ is a prime, and there is an odd prime $l$ such that $a b \equiv 0(\bmod l)$ and $e \equiv 0(\bmod 5)$, where $e$ is the order of $c$ modulo $l$, then equation (5) has only the solution $(x, y, z)=(2,2,5)$. 
In addition, Scott [15] proved a result which implies the following.

TheOREM E. If $c=2$, then the Terai-Jeśmanowicz conjecture is true. If $c$ is an odd prime, then there is at most one other solution to the TeraiJeśmanowicz conjecture.

In this note, we deal with the Terai-Jeśmanowicz conjecture for the special case $p=q=2, r$ odd. We have

Theorem. If $p=q=2,2 \nmid r, c \equiv 5(\bmod 8), b \equiv 3(\bmod 4)$ and $c \in \mathbb{P}^{\mathbb{N}}$, then the Terai-Jeśmanowicz conjecture holds.

Corollary 1 to Theorem. Let

$$
a=m\left|m^{2}-3 n^{2}\right|, \quad b=n\left|3 m^{2}-n^{2}\right|, \quad c=m^{2}+n^{2},
$$

where $m, n \in \mathbb{N}, \operatorname{gcd}(m, n)=1$. If $m \equiv 2(\bmod 4), n \equiv 1(\bmod 4)$ and $m^{2}+n^{2} \in \mathbb{P}^{\mathbb{N}}$, then equation (5) has only the solution $(x, y, z)=(2,2,3)$.

Corollary 2 to Theorem. Let

(6) $\quad a=m\left|m^{4}-10 m^{2} n^{2}+5 n^{4}\right|, \quad b=n\left|5 m^{4}-10 m^{2} n^{2}+n^{4}\right|, \quad c=m^{2}+n^{2}$, where $m, n \in \mathbb{N}$ with $\operatorname{gcd}(m, n)=1, m^{2}+n^{2} \in \mathbb{P}^{\mathbb{N}}$ and $m \equiv 2(\bmod 4)$. If one of the following cases holds, then equation (5) has only the solution $(x, y, z)=(2,2,5)$ :

CASE $1: m>\sqrt{2} n$ and $n \equiv 3(\bmod 4)$;

CASE 2: $m>\sqrt{10} n$;

CASE $3: n=1$.

From Corollary 1 , we see that if $m \equiv 2(\bmod 4)$ and $m^{2}+1 \in \mathbb{P}$, then the equation

$$
\left(m\left(m^{2}-3\right)\right)^{x}+\left(3 m^{2}-1\right)^{y}=\left(m^{2}+1\right)^{z}, \quad x, y, z \in \mathbb{N},
$$

has only the solution $(x, y, z)=(2,2,3)$.

2. Preliminaries. We will use the following lemmas.

LEMMA 1. If $2 \nmid r$ and $r>1$, then all solutions $(X, Y, Z)$ of the equation

$$
X^{2}+Y^{2}=Z^{r}, \quad X, Y, Z \in \mathbb{Z}, \operatorname{gcd}(X, Y)=1,
$$

are given by

$$
X+Y \sqrt{-1}=\lambda_{1}\left(X_{1}+\lambda_{2} Y_{1} \sqrt{-1}\right)^{r}, \quad Z=X_{1}^{2}+Y_{1}^{2},
$$

where $\lambda_{1}, \lambda_{2} \in\{-1,1\}, X_{1}, Y_{1} \in \mathbb{N}$ and $\operatorname{gcd}\left(X_{1}, Y_{1}\right)=1$.

Lemma 1 follows directly from a theorem in the book of Mordell [12], pp. 122-123. 
Lemma 2. For any $k \in \mathbb{N}$ and any complex numbers $\alpha, \beta$, we have

$$
\alpha^{k}+\beta^{k}=\sum_{j=0}^{[k / 2]}\left[\begin{array}{l}
k \\
j
\end{array}\right](\alpha+\beta)^{k-2 j}(-\alpha \beta)^{j},
$$

where

$$
\left[\begin{array}{c}
k \\
j
\end{array}\right]=\frac{(k-j-1) ! k}{(k-2 j) ! j !} \in \mathbb{N} \quad(j=0,1, \ldots,[k / 2]) .
$$

It is Formula 1.76 in [10].

Lemma 3. Let $a, b, c, p, q, r \in \mathbb{N}$ satisfy the hypotheses of the TeraiJeśmanowicz conjecture. If $p=q=2,2 \nmid r$, and if $c \equiv 5(\bmod 8), 2 \mid a$, then

$$
\left(\frac{a}{c}\right)=-1, \quad\left(\frac{b}{c}\right)=1,
$$

and so $2 \mid x$ in equation (5). Here $\left(\frac{*}{c}\right)$ denotes the Legendre-Jacobi symbol.

Proof. Since $p=q=2,2 \nmid r$, we have

$$
a^{2}+b^{2}=c^{r}, \quad a, b, c \in \mathbb{N}, \operatorname{gcd}(a, b)=1 .
$$

By Lemma 1, we deduce from (7) that

$$
a+b \sqrt{-1}=\lambda_{1}\left(m+\lambda_{2} n \sqrt{-1}\right)^{r}, \quad c=m^{2}+n^{2},
$$

where $\lambda_{1}, \lambda_{2} \in\{-1,1\}, m, n \in \mathbb{N}$ and $\operatorname{gcd}(m, n)=1$. From (8), we have

$$
\begin{aligned}
2 \lambda_{1} a & =\left(m+\lambda_{2} n \sqrt{-1}\right)^{r}+\left(m-\lambda_{2} n \sqrt{-1}\right)^{r}, \\
2 \lambda_{1} b \sqrt{-1} & =\left(m+\lambda_{2} n \sqrt{-1}\right)^{r}-\left(m-\lambda_{2} n \sqrt{-1}\right)^{r} .
\end{aligned}
$$

Hence, by Lemma 2 we have

$$
\begin{aligned}
\lambda_{1} a & =\frac{1}{2} \sum_{j=0}^{(r-1) / 2}\left[\begin{array}{l}
r \\
j
\end{array}\right](2 m)^{r-2 j}\left(-m^{2}-n^{2}\right)^{j} \\
& =m \sum_{j=0}^{(r-1) / 2}\left[\begin{array}{l}
r \\
j
\end{array}\right]\left(4 m^{2}\right)^{(r-1) / 2-j}\left(-m^{2}-n^{2}\right)^{j}, \\
\lambda_{1} b & =\frac{1}{2 \sqrt{-1}} \sum_{j=0}^{(r-1) / 2}\left[\begin{array}{l}
r \\
j
\end{array}\right]\left(2 \lambda_{2} n \sqrt{-1}\right)^{r-2 j}\left(m^{2}+n^{2}\right)^{j} \\
& =\lambda_{2} n \sum_{j=0}^{(r-1) / 2}\left[\begin{array}{l}
r \\
j
\end{array}\right]\left(-4 n^{2}\right)^{(r-1) / 2-j}\left(m^{2}+n^{2}\right)^{j} .
\end{aligned}
$$

Since $c \equiv 5(\bmod 8)$, and $2 \mid a$, we see from (8) and (9) that $2 \| m, 2 \nmid n$. 
So by (9) and (10), we have

$$
\begin{gathered}
\left(\frac{a}{c}\right)=\left(\frac{\lambda_{1} a}{c}\right)=\left(\frac{m\left(4 m^{2}\right)^{(r-1) / 2}}{m^{2}+n^{2}}\right)=\left(\frac{m}{m^{2}+n^{2}}\right)=-\left(\frac{m / 2}{m^{2}+n^{2}}\right)=-1, \\
\left(\frac{b}{c}\right)=\left(\frac{\lambda_{1} b}{c}\right)=\left(\frac{\lambda_{2} n\left(-4 n^{2}\right)^{(r-1) / 2}}{m^{2}+n^{2}}\right)=\left(\frac{n}{m^{2}+n^{2}}\right)=1 .
\end{gathered}
$$

This completes the proof of Lemma 3.

\section{Proof of the Theorem and its corollaries}

Proof of Theorem. Since $c \equiv 5(\bmod 8)$ and $b \equiv 3(\bmod 4)$, we have $2 \mid a$. By Lemma 3 , we find that $2 \mid x$. From $(5)$, we have $3^{y} \equiv 1(\bmod 4)$. Hence $2 \mid y$. Then by Theorem $\mathrm{D}$, we deduce that equation (5) has at most one solution $(x, y, z)$, except for

$$
(a, b, c)=(5,3,2),(13,3,2),(10,3,13) .
$$

Clearly, $(a, b, c) \neq(5,3,2)$ since $\max (a, b, c)>7$, and the equations $13^{p}+3^{q}=2^{r}$ and $10^{p}+3^{q}=13^{r}$ are all impossible since $p, q, r \geq 2$ (see [23] and [14]). Thus, (5) has only the solution $(x, y, z)=(2,2, r)$. The Theorem is proved.

Proof of Corollary 1. If $m>n / \sqrt{3}$, then we find that $b=n\left(3 m^{2}-n^{2}\right)$ $\equiv 3(\bmod 4)$. By the Theorem, Corollary 1 holds.

If $m<n / \sqrt{3}$, then

$$
a=m\left(3 n^{2}-m^{2}\right), \quad b=n\left(n^{2}-3 m^{2}\right), \quad c=m^{2}+n^{2} .
$$

By Lemma 3 and Theorem D, if $2 \mid y$, then Corollary 1 holds. Now assume that $2 \nmid y$. From (5),

$$
\left(\frac{n\left(n^{2}-3 m^{2}\right)}{3 n^{2}-m^{2}}\right)=\left(\frac{b}{3 n^{2}-m^{2}}\right)^{y}=\left(\frac{m^{2}+n^{2}}{3 n^{2}-m^{2}}\right)^{z} .
$$

Since $m \equiv 2(\bmod 4)$ and $n \equiv 1(\bmod 4)$, we have

$$
\begin{gathered}
\left(\frac{n\left(n^{2}-3 m^{2}\right)}{3 n^{2}-m^{2}}\right)=\left(\frac{n^{2}-3 m^{2}}{3 n^{2}-m^{2}}\right)=\left(\frac{3 n^{2}-m^{2}}{n^{2}-3 m^{2}}\right)=\left(\frac{8 m^{2}}{n^{2}-3 m^{2}}\right)=-1, \\
\left(\frac{m^{2}+n^{2}}{3 n^{2}-m^{2}}\right)=\left(\frac{3 n^{2}-m^{2}}{n^{2}+m^{2}}\right)=\left(\frac{-4 m^{2}}{n^{2}+m^{2}}\right)=1 .
\end{gathered}
$$

From (11)-(13), we get $-1=1$, a contradiction. This proves the corollary.

Proof of Corollary 2. From the Theorem, it suffices to prove Cases 2 and 3 of Corollary 2. Now we assume that

$$
a=m\left|m^{4}-10 m^{2} n^{2}+5 n^{4}\right|, \quad b=5 m^{4}-10 m^{2} n^{2}+n^{4}, \quad c=m^{2}+n^{2},
$$


$m>\sqrt{10} n$ and $n \equiv 1(\bmod 4)$. Clearly, $m^{4}-10 m^{2} n^{2}+5 n^{4} \in \mathbb{N}$. If $5 \nmid n$, then we have

$$
\begin{aligned}
& \left(\frac{b}{m^{4}-10 m^{2} n^{2}+5 n^{4}}\right) \\
& =\left(\frac{m^{4}-10 m^{2} n^{2}+5 n^{4}}{5 m^{4}-10 m^{2} n^{2}+n^{4}}\right) \\
& =\left(\frac{5}{5 m^{4}-10 m^{2} n^{2}+n^{4}}\right)\left(\frac{5 m^{4}-50 m^{2} n^{2}+25 n^{4}}{5 m^{4}-10 m^{2} n^{2}+n^{4}}\right) \\
& =\left(\frac{-40 m^{2} n^{2}+24 n^{4}}{5 m^{4}-10 m^{2} n^{2}+n^{4}}\right)=\left(\frac{5 m^{2}-3 n^{2}}{5 m^{4}-10 m^{2} n^{2}+n^{4}}\right) \\
& =\left(\frac{5 m^{4}-10 m^{2} n^{2}+n^{4}}{5 m^{2}-3 n^{2}}\right)=\left(\frac{-7 m^{2}+n^{2}}{5 m^{2}-3 n^{2}}\right) \\
& =\left(\frac{5}{5 m^{2}-3 n^{2}}\right)\left(\frac{-7\left(5 m^{2}-3 n^{2}\right)-16 n^{2}}{5 m^{2}-3 n^{2}}\right)=\left(\frac{5 m^{2}-3 n^{2}}{5}\right)=-1 .
\end{aligned}
$$

If $5 \mid n$ then we also have

$$
\left(\frac{b}{m^{4}-10 m^{2} n^{2}+5 n^{4}}\right)=-1
$$

by a similar method. Moreover

$$
\left(\frac{c}{m^{4}-10 m^{2} n^{2}+5 n^{4}}\right)=\left(\frac{m^{4}-10 m^{2} n^{2}+5 n^{4}}{m^{2}+n^{2}}\right)=\left(\frac{16}{m^{2}+n^{2}}\right)=1 .
$$

Hence, from (5) we have $2 \mid y$. From Lemma 3 we deduce similarly that $2 \mid x$. Then Theorem D implies Case 2. For Case 3 the only remaining case is $m=2$. Then $a=38, b=41, c=5$. As above we find $2|x, 2| y$, and Case 3 follows from Theorem D.

4. An open problem. Let $r>1$ be a given odd number, and let

$$
\begin{aligned}
a & =m\left|\sum_{j=0}^{(r-1) / 2}\left[\begin{array}{l}
r \\
j
\end{array}\right]\left(4 m^{2}\right)^{(r-1) / 2-j}\left(-m^{2}-1\right)^{j}\right|, \\
b & =\left|\sum_{j=0}^{(r-1) / 2}\left[\begin{array}{l}
r \\
j
\end{array}\right](-4)^{(r-1) / 2-j}\left(m^{2}+1\right)^{j}\right|, \\
c & =m^{2}+1,
\end{aligned}
$$

where $m \in \mathbb{N}$ with $m^{2}+1 \in \mathbb{P}$ and $m \equiv 2(\bmod 4)$. Clearly, $(a, b, c)$ is a solution of equation (7). Is it possible to prove the Terai-Jeśmanowicz 
conjecture by the method of this paper under the above condition? When $r=3,5$, the answer to the question is "yes".

Acknowledgements. The author would like to thank the referee for his valuable suggestions.

\section{References}

[1] Z. F. Cao, On the Diophantine equation $a^{x}+b^{y}=c^{z}$, I, Chinese Sci. Bull. 32 (1987), 1519-1521; II, ibid. 33 (1988), 237 (in Chinese).

[2] - On the Diophantine equation $a x^{2}+b y^{2}=p^{z}$, J. Harbin Inst. Tech. 23 (1991), 108-111.

[3] -, Divisibility of the class numbers of imaginary quadratic fields, Acta Math. Sinica 37 (1994), 50-56 (in Chinese).

[4] —, On Jeśmanowicz' conjecture, Res. Rep. Harbin Inst. Tech. 253 (1982), 1-14.

[5] -, Introduction to Diophantine equations, Harbin Inst. Tech. Press, 1989 (in Chinese).

[6] Y. D. Guo and M. H. Le, A note on Jeśmanowicz' conjecture concerning Pythagorean numbers, Comment. Math. Univ. St. Paul. 44 (1995), 225-228.

[7] T. Hadano, On the Diophantine equation $a^{x}=b^{y}+c^{z}$, Math. J. Okayama Univ. $19(1976 / 77), 25-29$.

[8] M. H. Le, On Jeśmanowicz' conjecture concerning Pythagorean numbers, Proc. Japan Acad. Ser. A Math. Sci. 72 (1996), 97-98.

[9] —, A note on Jeśmanowicz' conjecture, Colloq. Math. 69 (1995), 47-51.

[10] R. Lidl and H. Niederreiter, Finite Fields, Addison-Wesley, Reading, MA, 1983.

[11] A. Mąkowski, On the diophantine equation $2^{x}+11^{y}=5^{z}$, Nord. Mat. Tidskr. 7 (1959), 81-96.

[12] L. J. Mordell, Diophantine Equations, Academic Press, 1969.

[13] T. Nagell, Sur une classe d'équations exponentielles, Ark. Mat. 3 (1958), 569-582.

[14] M. Perisastri, A note on the equation $a^{x}-b^{y}=10^{z}$, Math. Student 37 (1969), 211-212.

[15] R. Scott, On the equations $p^{x}-b^{y}=c$ and $a^{x}+b^{y}=c^{z}$, J. Number Theory 44 (1993), 153-165.

[16] Q. Sun and X. M. Zhou, On the Diophantine equation $a^{x}+b^{y}=c^{z}$, Chinese Sci. Bull. 29 (1984), 61 (in Chinese).

[17] K. Takakuwa, On a conjecture on Pythagorean numbers, III, Proc. Japan Acad. Ser. A Math. Sci. 69 (1993), no. 9, 345-349.

[18] K. Takakuwa and Y. Asaeda, On a conjecture on Pythagorean numbers, ibid. 69 (1993), no. 7, 252-255.

[19] —, -, On a conjecture on Pythagorean numbers, II, ibid. 69 (1993), no. 8, 287-290.

[20] N. Terai, The Diophantine equation $a^{x}+b^{y}=c^{z}$, ibid. 70 (1994), 22-26.

[21] - , The Diophantine equation $a^{x}+b^{y}=c^{z}$, II, ibid. 71 (1995), 109-110.

[22] - The Diophantine equation $a^{x}+b^{y}=c^{z}$, III, ibid. 72 (1996), 20-22.

[23] S. Uchiyama, On the Diophantine equation $2^{x}=3^{y}+13^{z}$, Math. J. Okayama Univ. 19 (1976/77), 31-38. 
[24] X. Z. Yang, On the Diophantine equation $a^{x}+b^{y}=c^{z}$, Sichuan Daxue Xuebao 4 (1985), 151-158 (in Chinese).

Department of Mathematics

Harbin Institute of Technology

Harbin 150001, P.R. China

E-mail: zfcao@hope.hit.edu.cn

Received on 21.9.1998

and in revised form on 7.5.1999 